\title{
The Miraculous Left Hand - On Leonardo da Vinci and the Search for a Common Understanding of Man and Nature
}

\author{
JENS ERIK FENSTAD \\ University of Oslo, Ovenbakken 11, 1361 Osteras, Norway. \\ E-mail: jfenstad@math.uio.no
}

Is a common approach to knowledge about man and nature possible? With Leonardo da Vinci as our starting point we will explore this question. Leonardo was much more than a painter; he was a sharp observer of man and nature. What he saw (structures) and what he did (using his miraculous left hand as syntax) was translated into insights and methods of constructions as recorded in his notebooks. His view was holistic, as witnessed by his study of the eye; from the anatomy of the eye he proceeded outwards to a theory of perspective, and inwards to an understanding how perception and the mind are grounded in the brain. In this we see similarities to current studies of language, mind and brain. It is possible to see a kind of proto-version of the art and science of mathematical modelling in Leonardo. This is a methodology of wide scope, extending far beyond physics and engineering. With a sufficiently broad understanding of the key concepts of structure, syntax and algorithms we have a method strong enough to allow for a common approach to knowledge of man and nature, to what there is and how we know.

Is a common understanding of man and nature possible? Science, in the broader sense of 'Wissenshaft', seems to say no, negating any attempt by man to achieve a unified view of knowledge. What we observe is a science progressing in many, perhaps even contradictory directions. Many would insist that this is as it should be. Science is a growing body which cannot be constrained by any aprioristic scheme of thought; the unity of science is and should be problematic. From any local point of view this may be true. But in a broader historical and cultural perspective we see traditions where different disciplines have interacted to create a common environment of learning and practice. And it is the experience of such cultural traditions that has justified a belief in the unity of knowledge.

We may point to one dominant tradition in our Western culture where mathematics, philosophy and our understanding of nature came together in a broad stream, flowing from Antiquity to Kant and Laplace. This tradition had an enormous influence; we cannot, to 
mention one important example, understand the Cartesian methodology, its range and limitations, without at the same time having an insight into Descartes' analytic geometry and mechanistic physics. And the mathematical framework of this development had an important impact on the general theory of knowledge. It informed, but it also set limits, not always understood, on our understanding of key concepts such as determinism and causality as seen in processes in both nature and society.

Another tradition is represented by the growing understanding of man as a biological species, covering the development from Darwin to current population genetics and molecular biology. This is a second example where we see many disciplines and traditions coming together, and it is only through a sound insight into this historical development that we can judge both the strength and the limitations of this process. Too many people are misled by the grand words and splendid rhetorics of vulgar Darwinism and become easy prey to the social Darwinism of earlier times and the sociobiology of today.

However, an understanding of the unity of knowledge requires more than physics and biology. It must also 'explain' man as a thinking individual, as a carrier of meaning and consciousness.

The cognitive side was not entirely absent from the earlier history. Let us take grammar as our example. A knowledge of grammar and logic was an important part in the first stages of the development from Antiquity to Kant. But this did not last. We observe that the Grammatica Speculativa ${ }^{1}$ of Thomas of Erfurt from 1315, a strong brew of language, logic and philosophy, has disappeared from our shared historical consciousness, as has the grammar of the Jansenists of Port Royal, the latter known as the refuge of Pascal. We remember only a 'royal list' of natural scientists, Euclid, Ptolemy, Copernicus, Kepler, Galileo, and Newton. But the situation is changing. The science of language underwent a major development starting with the Dane Rasmus Rask and the comparative linguistics of the mid nineteenth century, ${ }^{2}$ continuing with de Saussure and structuralism from the beginning of the twentieth century, then the theoretical linguist Chomsky in the middle part of that same century, to the developments in language and speech technology that we see today. ${ }^{3}$ For many people language is the unique characteristics of man as a biological species; thus, if the current science and technology is successful, this should offer some promise of including the study of man and meaning in the broader science of man as part of nature. Let only the molecular biologists and the speech technologists get on with their work.

Not everyone would agree. In the same way as the chess-playing machine is not a human being, a speech-generating machine is not an individual with consciousness and the capacity for meaningful language. Thus, for many the initial question mark will stand. Mind and body belong to different categories, for how could my consciousness and my understanding of myself be captured by any combination of measurements of time, length, and mass. The humanities and the natural sciences, both of equal importance, must in their methods go their separate ways. The dream of a common way of understanding has, however, deep roots in our culture. Much has been written and much can still be said about this, and the literature is enormous. But I note, with some surprise, that there is one man who stood right at the beginning of the quest for a common understanding, and who is often not given proper recognition: Leonardo da Vinci. 
To the general public Leonardo da Vinci is primarily known for the Mona Lisa, a high point in Western art. Less known is the scientist Leonardo and his view on the nature and unity of knowledge. ${ }^{4}$

\section{Leonardo da Vinci}

Leonardo's formative years were spent in the workshops and studios in Firenze as an apprentice in many trades. He was left-handed, a not uncommon phenomenon among men of talents, and his 'miraculous left hand' made it an easy task for him to reproduce in drawings and paintings what he was working on, and more generally, what he observed. In the earlier stage, as an apprentice, we see mere reproductions in his notebooks. But with experience and practice he moved beyond, and many new inventions of his own started to make their appearance. He did not have a mathematical way of expressing his thoughts and observations, as did the astronomers of Antiquity and the scientists of the later Renaissance period; he observed and he reproduced what he saw. Water and systems of water supply were an important domain of activity for the young technologist Leonardo, and in the notes he left we find several sharp observations of hydrological processes. But despite the many achievements of Archimedes, the time was not ripe for a mathematical analysis of 'real' hydrological phenomena. Later, Leonardo's interests expanded to include natural processes in geology and living nature, the latter also including man's body and soul. Gradually, he developed a vision how to understand the interconnectedness of things. He wanted in the same framework to obtain an insight into the mechanical principles behind the many inventions and tools of the engineer, and into the corresponding 'mechanical' principles governing processes in nature and man. His studies of how water flowed inspired him to search for a more general theory of how objects and forms are transformed. Again he lacked a mathematics strong enough to describe what he 'saw', but in several series of sketches from his notebooks we can, with generous hindsight, see the beginning of a geometrical transformation theory. ${ }^{5}$

To 'see' was the method of Leonardo, and in this he made a break with the traditional learned style of the Renaissance, where interpretation of the newly discovered texts from the classical Greek period was the chosen method; we recall many illustrations in the medical texts from the anatomy theatres of the Renaissance where the learned professor reads from an ancient text, perhaps from a recovered manuscript of Galen, while at the same time the assistant performs a dissection on a body on a table in front of the professor. For the learned man the link between text and reality, in this case a human cadaver, was rather weak, what was 'seen' and what was 'read' were not necessarily connected. Leonardo represented something new and different. Knowledge for him was always grounded in what was 'seen'. This was a break with a long-lasting tradition. It is of interest to note that even Galileo, a century later, was in many ways a follower of the old tradition, where an 'experiment' more often was a literary illustration of an accepted truth than something immediately 'seen'. ${ }^{6}$ Returning to Leonardo, we note that the anatomy of the eye became a high point in his studies of man. He followed his anatomical investigations of the eye outwards, from the primary fact of seeing to a search for a structure for what was seen. And in this process he created a new theory of perspective to 
serve as a foundation for his art and more generally for his understanding of nature. He also pursued his studies of the eye inwards into the brain to search for an anatomical foundation for man's emotions and cognitive abilities. And the 'outwards' and the 'inwards' should be connected. Leonardo as scientist moved far beyond the painter described by current tradition. And different from the later Descartes, Leonardo was no dualist making a sharp distinction between body and mind. ${ }^{7}$ His work can be read as an argument for the unity of the two, with a mind firmly grounded in the anatomy of the brain.

Later times have not always seen Leonardo in this perspective. It is true that some enthusiastic supporters of Leonardo the scientist, such as the French philosopher of science and historian Pierre Duhem, have given Leonardo a prominent role in the development of modern science. More sober observers, such as the well known historian of science E.J. Dijksterhuis, remained unimpressed. In his major study Die Mechanisierung des Weltbildes he very briefly notes that Leonardo was of almost no importance compared with the great names of the scientific revolution. ${ }^{8}$ Today we allow for a somewhat more generous view. Leonardo's originality as engineer is convincingly documented in P. Galluzzi's rich book Renaissance Engineers from Bruelleschi to Leonardo da Vinci, from $1996 .{ }^{9}$ Galluzzi, who is Director of the Istituto e Museo di Storia della Scienza in Firenze, writes with rare insight and authority about Leonardo. Leonardo's science has also been the subject of two recently published books, The Science of Leonardo by Capra in 2007 and Leonardo's Legacy by Klein in 2010. ${ }^{10}$ Both applaud, perhaps a bit too enthusiastically, the scientist and his attempts to understand complex systems, and both deplore the lacking recognition of Leonardo as a pioneer scientist. But the latter neglect may have a simple explanation. Later times knew Leonardo only as painter, his notebooks and drawings for the longest time went unpublished. Thus, it is Andreas Vesalius who has been recognized in the standard histories of medicine as the creator of modern anatomy, whereas the pioneering work of Leonardo of equal importance was hidden in his - as good as forgotten - notebooks. ${ }^{11}$

Leonardo was much more than a painter and illustrator though. He therefore merits an honourable mention in the history of science supplementing the 'royal list' of the scientific revolution. But we should also be aware of his limitations. We may rightfully recognize that his morphology for complex structure was remarkable at the time, but today his notebooks and drawings are mostly of historical interest, and not a working source for current science. This is, however, not the end of the story. If the artist Leonardo and the scientist Leonardo had been part of a living memory today, C.P. Snow's book on the two cultures need not have been written.

\section{What did Leonardo 'See'?}

To meet for the first time the sketches and paintings of Leonardo is a powerful experience. It is far beyond my competence to describe and analyse his art in any detail; the reader would be well advised to consult the sources I have mentioned in the section above. But a mathematician may have something different and new to add to the standard expositions of what Leonardo 'saw'.

Mathematics often appears to the common man as a vast collection of numbers, equations and complex geometrical constructions, to be learnt on command and to be 
applied with more or less skill in everyday tasks of counting and measurements. But mathematics is not what the common man believes, as I will try to explain. A key concept necessary for understanding mathematics is the notion of structure. ${ }^{13}$ To be brief, a structure consists of two parts. One is the domain of the structure, which is some given collection of things, objects, or individuals of any kind, be they of material, mental or cultural origin. The second part consists partly of properties that the objects are supposed to possess, and partly of relations these objects bear to each other. 'Common man' will correctly suspect that this is how a mathematician writes, and it is true that the simplest examples of structures come from mathematics, but the concept has, as later parts of this essay will show, a much vaster range, not only including mathematics and the natural sciences, but also art and the humanities.

One simple example of a structure is the collection of natural numbers $0,1,2,3, \ldots$, where the defining relations are addition and multiplication; some basic defined properties are even and odd and being a prime number. Essential for all kinds of measurements and construction are geometrical structures based on points, lines and surfaces. And for such structures length and distance are fundamental properties and relations. School mathematics knows its geometry through the heritage from Euclid's Elements with its characteristic axiomatic approach. But geometry was not only an intellectual activity of the learned class, the artisans and the master builders knew their geometry as part of their trade, as the cathedrals of the medieval ages bear witness.

It was to this latter tradition Leonardo belonged. Indeed, it was in his early years as apprentice in the workshops in Firenze that he got a 'grasp' of what structures in essence are. We are impressed by his sketches and notes on running water and on many kinds of mechanisms connected to waterworks. We are equally impressed by his studies of geological forms and structures; sometimes in his paintings he seems to pay even more attention to the landscape and the mountains in the background than to the people in the front. And we note that the structures represent Leonardo's own interpretation of what he 'sees', when he for example does not give the 'correct' reproduction of light and shadows, but uses the shadows to open up for a deeper insight into the character of the person he is painting. The interested reader should turn to the discussion in Klein ${ }^{10}$ of Raphael's Portrait of a Lady with a Unicorn compared with Leonardo's Mona Lisa.

To give what is 'seen' a structure was for Leonardo a first step toward insight and eventual applications. He was part of an artisan tradition that lived side by side with an emerging mathematical natural science and technology. For the role this artisan tradition played within engineering I recommend the book Engineering and the Mind's Eye by Ferguson. ${ }^{14} \mathrm{He}$ shows through a series of examples how engineering practice traditionally, and even today, goes well beyond the purely scientific calculations possible at the time and included qualities such as how structures are 'seen' and how qualities of materials are judged, i.e. properties solidly within the domain of the Mind's Eye. I would guess that boat designers of today would still agree to this, even if computers are fast approaching. For an example from the history of natural science I refer to the study Image and Reality by Rocke, ${ }^{15}$ which convincingly documents how a visualization of chemical structures in the mid-nineteenth century, above all as seen in the work of the German chemist A. Kekulé, started a structural revolution going well beyond what 'official' theory of that time could sanction. 


\section{The Miraculous Left Hand}

The structures seen by Leonardo were not only structures frozen in time. Many examples from his notebooks show structures undergoing a temporal development, in particular, the many examples he drew from hydrodynamics and geology. But the act of 'seeing' structures is only the first step on the road to a complete understanding and to a knowledge necessary to make use of them. To be able to manipulate and control structures we need an appropriate language or syntax. It was exactly on this point that the 'royal list' of Kepler, Galileo and Newton, made a decisive break-through in our understanding of nature. Their glory was to create a mathematical description of how bodies move, valid both for planets in the sky and cannonballs on earth. To appreciate this it is important to observe a separation between syntax and structure. Galileo's equations for falling bodies, Kepler's equations for the orbit of planets, and the laws of force written down by Newton are linguistic expressions for what is 'seen' in nature. And this mathematical formalism also included a description of the long-term time development of the structures, thus allowing us to predict when a spacecraft will land on the moon, or how to aim our cannons against a foreign fortress. It is important to stress that syntax presupposes structure. The equations we write down, are always an expression of some properties of the structures 'seen'. Thus, if structure is changed, as for example when we move from classical to relativistic mechanics, syntax including rules and equations must also be rewritten.

The syntax Leonardo used was in a special way embedded in his 'miraculous left hand'. He could not, as Galileo did, write the equations for a ball rolling down an inclined plane. But he could reproduce through a connected series of sketches both the static and dynamic properties of what he observed. And true to his background as craftsman and engineer it was the everyday and complex phenomena that caught his attention. Leonardo had a strong interest in mathematics, but a mathematician he never became despite many tutorials from close mathematical friends. We must also, in order to be fair towards him, admit that a mathematical syntax for the kind of phenomena that interested him, was of such a complexity compared to the pendulum and the inclined planes of Galileo, that we only today can see the beginning of a satisfactory analysis. But the eye of Leonardo was so sharp that 'he grasped connections using nothing more than his pencil that it took other scientists many generations to formulate in equations' ${ }^{16,24}$ This observation (see Ref. 24, p. 181) by a well respected professor of bioengineering at Caltech, necessitates some further remarks on mathematics, or rather on the combined art and science of mathematical modelling. We must then add a new concept to the familiar pair of structure/syntax; this is the notion of the algorithm. The act of modelling has three stages: first, an analysis of a given scientific or technological problem; through this analysis we are led to a suitable structure of the problem. Next, the determination of an appropriate syntax, including equations or more generally rules, in order to describe and analyse the structure. Finally, the development of algorithms, today mostly given in the form of (computer) programmes, which in a scientific context can be used to predict later stages of the evolution of the structure, and which in technological applications can be used to provide blueprints for construction and production. 
But equations have their limitations: we have learned that it is only in very special cases that they are exactly solvable. In all other cases we have to resort to simulations and graphical representations of the solutions. This represents in many ways a 'third way' to understand nature, where the first two are theory and experiments. Climate and environment offer good examples. We do have, at least in principle, the correct equations from hydrodynamics. Starting from these equation, Vilhelm Bjerknes wrote down in the first years of the nineteenth century a set of equations for weather prediction. Bjerknes was guided by a deep insight into the structure and syntax of the phenomenon he studied. But his equations were complex (necessarily of non-linear nature) and could only be applied through simulations and graphical representations. ${ }^{17}$ And it is at this point that we see a link back to Leonardo. He had a good command of two of the parts of mathematical modelling, structure and graphical representations. He lacked a mathematical syntax, hence the somewhat negative remark from Dijksterhuis ${ }^{18}$ on the limited importance of Leonardo for the development of the mechanical sciences. But what Leonardo did points, as we shall in the next section, towards a broader applicability.

Reviewing the three basic concepts structure, syntax and algorithms, we come to understand how the general notion of structure, as described above, and the extended notion of an algorithm or programme, well-known to everyone who ever used a computer, also extend our possibilities for choice of syntax. It is therefore possible today to proceed with this mode of analysis far beyond the equations of physics, perhaps even to such core areas of the humanities as language and meaning. We shall in the final section reflect on what this implies for our search for a common understanding of man and nature.

\section{A Common Understanding}

Leonardo was no dualist. Based on his analysis of the anatomy of the eye and how it functioned, the soul was for him in a direct and concrete way located in the brain. He observed, he made sketches and notes, but despite his deep understanding of the complexity of nature, this was but suggestions and beliefs. The search for a common understanding was still a distant goal.

Science progresses through doing what is doable. The winning strategy of the 'royal list' was to simplify, to abstract from the full complexity of nature and to single out some specific aspects that could be studied in depth. Thus, Galileo restricted himself to observing running balls on an inclined plane where Leonardo aimed at a full understanding of the behaviour of 'real' water in actual rivers and waterworks. The simplifying strategy won the day, and modern natural science grew out of the simple equations written down by Galileo, Kepler and Newton. The main ingredients became experiments, analysis and synthesis. And the strategy for the understanding of more complex situations would first be to break down a complex problem into its simplest component parts and to study these parts in isolation, then as the next step to determine how the overall behaviour was a well-defined function of the operations of the component parts. It is not surprising that the success of this approach led to a wide-spread belief in a general reduction of man and nature to nature's basic parts, i.e. to physics and physics alone. As late as 1980 we find a robust and almost unbelievable expression of this view in the 
Inaugural Lecture ${ }^{19}$ delivered by Stephen Hawking when accepting the professorship once held by Newton. And true to this heritage he started out by affirming his strong belief in a final and complete theory of physics. This was soon to be expected; then in orderly and neat succession would follow first a reduction of chemistry, next of biology, and finally of the study of human behaviour to the basic equations of physics. Hawking had to admit that the programme was not yet completed, he did with some regret note that 'although in principle we know the equations that govern all of biology, we have not been able to reduce the study of human behaviour to a branch of applied mathematics'. Thirty years have passed since Hawking gave this lecture, and I doubt if he would have said exactly the same today. Both science and technology have progressed beyond the limits enforced by the traditional use of linear equations; we now see the beginnings of a richer mathematics necessary for a deeper understanding of non-linearity and causal interactions in complex systems.

Let us one more time return to Leonardo. His struggle to understand nature lost out compared with the strategy of Galileo, Kepler and Newton. But those who won had a prize to pay, their world became limited and colourless. And they were committed to a reductionist strategy, as seen in the quote from Hawking above. But despite these limitations we have to admit that the reductionist strategy seen in a historical perspective was a great success and became a fundamental driving force in the growth of Western society. However, in the attempt to understand the interaction between body and mind, problems arose, and it is not surprising that some form of dualism became a preferred way out. If you cannot construct the soul and human consciousness from what exists at the atomic level and the forces active there, the soul must have another mode of existence. This was what Leonardo denied. His view was holistic and not reductionist. The structures he 'saw' were meant to represent the phenomena in their full complexity, both 'outwards' in nature and 'inwards' in the brain. I do not in any sense argue that Leonardo's way should have replaced the methodology of Galileo, but I do suggest that if we had let both live and interact, we would have inherited a richer view on the relationship between man and nature; C.P. Snow's book on the two cultures, ${ }^{12}$ as noted above, would have been unnecessary.

The interaction between language, meaning and brain will be our counterexample to criticism of Snow. The challenge is to understand how language and meaning can be grounded in the dynamical activity of large and complex systems of neurons in the brain. The study of language must necessarily proceed on many levels. At the 'top level' we need a theory of grammar including both syntax and semantics, at the 'bottom level' we need a detailed understanding of the structure and the functioning of the brain. And 'top' and 'bottom' must be linked. To achieve this we need more than the notes and sketches of Leonardo.

And much has indeed happened in linguistics and the connected cognitive sciences. ${ }^{20}$ New and powerful experimental and theoretical methods have been developed to help understand both linguistic and mental phenomena. We have, in particular, seen an increased use of mathematics and the natural sciences in this endeavour; mathematical modelling in the sense described above has become a standard tool. But despite this tremendous step forward we are still in a somewhat uncertain state in our understanding of the gap between language and brain. Many ways of closing this gap have been proposed. 
There is a clear connection between our sensual perceptions, our ability to see, hear, feel and touch, and the dynamical processes in our brain. There are also connections between grammatical categories and human perceptions. If these insights are added together with the necessary use of some mathematics, it is possible to suggest a link between language and brain. ${ }^{21}$ This is just one example of how current science tries to ground cognitive functions in the brain. But one should show proper caution. In this area there is no lack of great visions and speculations on how mind and body are linked together in one grand holistic system, but in almost all such cases this is exactly what it is, speculation and not 'hard' science.

This is not necessarily the end of the story. Despite our sceptical attitude there seem to be sufficient grounds to claim that language and meaning are grounded in the brain. It is important here to have a clear idea of what we mean by the term 'grounded'. We have gradually come to understand the notion of causality in complex systems and the limits for classical reductionism in non-linear systems. In such systems a complete theory necessitates explanations on many levels and an understanding of causal interaction between levels; ${ }^{22}$ contra Hawking there is more than a single 'upward' causal chain from physics to mind and culture. The interaction of levels does not preclude that levels may have a large degree of autonomy, and that a satisfactory explanation of a problem or phenomenon can be given within a single level. Let our example be the linguistic analysis of metaphors; hardly anyone would insist that the equations needed to understand the dynamics of the brain should be present in every linguistic analysis of metaphors. Our understanding of man, meaning and nature will therefore not be a simple-minded reductionistic explanation, but could also be an understanding of phenomena at their own appropriate level; the student of literature need not start every analysis with a section on brain structure. But in the same way as all properties of thermodynamics are grounded in the molecular level and what there exists, every analysis of metaphors and meaning is necessarily linked to the human brain; the sceptical reader is well advised to turn to the discussion in P. Gärdenfors' book, Conceptual Spaces; The Geometry of Thought. ${ }^{23}$

Our discussion of Leonardo pointed in two directions, to what he saw and to what he did. As to the first dimension, which is the level of structures, I would argue that we clearly see similarities between our discussion of language, meaning and brain and Leonardo's studies, both 'outwards' and 'inwards', of the eye. The second dimension, represented by syntax and algorithms, was present in his 'miraculous left hand'. It is thus possible to see some 'proto-version' of the art and science of mathematical modelling in Leonardo's way of work. Such modelling is a methodology of wide scope; in the discussion above on language and brain we showed how this method now extends to fields far beyond physics and engineering. With a sufficiently broad understanding of the key concepts of structure, syntax and algorithm we have a method strong enough to allow for a common approach to knowledge of man and nature, to what there is and how we know.

\section{Notes and References}

1. Thomas' book is reprinted together with an English translation in G.L. Bursill-Hall (1972) Grammatica Speculativa of Thomas of Erfurt (London: Longman). For a broader analysis of medieval grammar see G.A. Paley (1976) Grammatical Theory in Europe 1500-1700. The Latin Tradition (Cambridge: Cambridge University Press). 
2. H. Pedersen (1972) The Discovery of Language. Linguistic Science in the 19th Century (Indiana University Press) is our source for Rasmus Rask and linguistics in the nineteenth century.

3. N. Chomsky (1957) Syntactic Structures (The Hague: Mouton.Chomsky. I have given my view of the development of linguistics from Thomas of Erfurt to Chomsky and the language technology of today in J.E. Fenstad (2010) Grammar, Geometry, and Brain (Chicago: University of Chicago Press).

4. The literature on Leonardo is extensive; an easily accessible biography is C. Nicholl (2005) Leonardo da Vinci, The Flights of the Mind (London: Penguin Books).

5. See the chapter 'Geometry done with motion' in F. Capra (2007) The Science of Leonardo (New York: Anchor Books).

6. Here I touch upon a topic which is extensively discussed in P. Dear (1995), Disciplines and Experience. The Mathematical Way in the Scientific Revolution (Chicago: University of Chicago Press).

7. For an analysis of the dualism of Descartes from the perspective of current cognitive science see D.M. Clarke (2003) Descartes' Theory of Mind (Oxford: Clarendon Press).

8. The sources are: P. Duhem (1906-1913) Etudes sur Leonardo de Vinci, 3 vols (Paris: Hermann) and E.J. Dijksterhuis (1956) Die Mechanisierung des Weltbildes (Berlin: Springer Verlag). I recommend A.R. Turner (1995) Inventing Leonardo. The Anatomy of a Legend (London: Papermac) for a critical review of the changing conceptions of Leonardo and his work.

9. P. Galluzzi (1996) Renaissance Engineers. From Brunelleschi to Leonardo da Vinci (Florence: Giunti).

10. F. Capra (2007) The Science of Leonardo (New York: Anchor Books); S. Klein (2010) Leonardo's Legacy (Cambridge, MA: Da Capo Press).

11. A.G. Debus (1978) Man and Nature in the Renaissance (Cambridge: Cambridge University Press). Debus comments at length on Vesalius, Leonardo gets just one sentence: Leonardo da Vinci's (1452-1519) earlier anatomical drawings were masterful, but unfortunately of little impact as they were unpublished.

12. C.P. Snow (1959) The Two Cultures (Cambridge: Cambridge University Press).

13. The most general analysis of the notion of structure is today found in mathematical logic. This analysis has an important root in the work of the Norwegian logician Thoralf Skolem, see J.E. Fenstad and H. Wang (2009) Thoralf Albert Skolem. In Handbook of the History of Logic, vol 5 (Amsterdam: North-Holland). But the logic approach may at times be somewhat too restrictive. An alternative would be to see the notion of structure in light of prototype theory, which in a sense is to understand structure as a kind of 'fixed point' for what is seen; see E. Rosch (1978) Prototype classification and logical classification. In: E. Scholnik (ed) New Trends in Cognitive Representations (Hillsdale, NJ: Lawrence Erlbaum Associates) and P. Gärdenfors (2000), Conceptual Spaces, The Geometry of Thought (Cambridge, MA: MIT Press), see also the discussion in J.E. Fenstad (2010) Grammar, Geometry, and Brain (Chicago: University of Chicago) pp. 45-46.

14. E.S. Ferguson (1993) Engineering and the Mind's Eye (Cambridge, MA: MIT Press).

15. A.J. Rocke (2010) Image and Reality, Kekulé, Kopp and the Scientific Imagination (Chicago: University of Chicago Press).

16. A current example of this can be found in C. Eloy (2011) Leonardo's rule, selfsimilarity and wind-induced stresses in trees. Physics Review Letters, 107, pp. 258101-258105. Eloy writes down the necessary equations to explain Leonardo's observation that the total cross-section of a tree remains the same along its height every time it branches. 
17. This is for our purposes an important example. Bjerknes and the Bergen school in meteorology, with its root both in the practise of actual weather forecasting and in the physics of hydrodynamical phenomena, got the two first parts correct, i.e. the structures and the equations, but it was only with the advent of modern computing around 1950 that the last part came into existence and algorithms were developed and implemented to enable real-time weather prediction. Numerical weather forecasting was an early example of mathematical modelling based on structure, syntax and algorithms; see K.C. Harper (2008) Weather by the Numbers.

The Genesis of Modern Meteorology (Cambridge, MA: The MIT Press) for a full account.

18. See E.J. Dijksterhuis (1956) Die Mechanisierung des Weltbildes (Berlin: Springer Verlag). Note that Dijksterhuis judges Leonardo totally in terms of classical mechanics, non-linearity and complexity had not yet reached the science historians around 1950.

19. S. Hawking (1980) Is the End in Sight for Theoretical Physics?An Inaugural Lecture (Cambridge: Cambridge University Press).

20. For a survey see R. Jackendoff (2002) Foundations of Language (Oxford: Oxford University Press); C.M. Brown and P. Hagoort (eds) (1999) The Neurocognition of Language (Oxford: Oxford University Press) and J.E. Fenstad (2010) Grammar, Geometry, and Brain (Chicago: University of Chicago Press).

21. J.E. Fenstad (1998) Formal semantics, geometry and mind. In: X. Arrazola et al. (eds) Discourse, Interaction and Communication (Amsterdam: Kluwer).

22. See G. Ellis (2005) Physics, complexity and causality. Nature, 435; K.N. Laland, J. Odling-Smee and S. Myles (2010) How culture shaped the human genome: bringing genetics and the human sciences together. Nature Reviews/Genetics, 11.

23. P. Gärdenfors (2000) Conceptual Spaces, The Geometry of Thought (Cambridge MA: MIT Press).

24. S. Klein (2010) Leonardo’s Legacy (Cambridge MA: Da Capo Press).

\begin{abstract}
About the Author
Jens Erik Fenstad is Professor Emeritus of Mathematics in the University of Oslo. He was Chairman of the Standing Committee for the Physical and Engineering Sciences of the ESF, a former President of the International Union of History and Philosophy of Science, and past Chair of the UNESCO World Commission on the Ethics of Scientific Knowledge and Technology.
\end{abstract}

